\title{
Ensayos a flexión de losas de concreto sobre terreno reforzadas con fibras de acero
}

\section{Flexural Tests of Concrete Slabs-on-Ground Reinforced with Steel Fibers}

\author{
Carrillo Julián \\ Universidad Militar Nueva Granada, UMNG, Colombia \\ Departamento de Ingeniería Civil \\ Correo:wjcarrillo@gmail.com
}

\author{
Silva-Páramo Diego \\ Universidad Militar Nueva Granada, UMNG, Colombia \\ Departamento de Ingeniería Civil \\ Correo:diego.silva@gmail.com
}

Información del artículo: recibido: junio de 2015, reevaluado: noviembre de 2015, aceptado: enero de 2016

\section{Resumen}

El concreto reforzado con fibras de acero (CRFA) se ha convertido en una solución para mejorar el desempeño del concreto y optimizar los sistemas constructivos. En este artículo se presentan y discuten los resultados de un programa experimental para estudiar el desempeño a flexión de losas de concreto sobre terreno reforzadas con fibras de acero para uso en vivienda. El programa experimental incluyó ensayos a flexión de ocho losas cuadradas de concreto de $600 \mathrm{~mm}$ de lado y $100 \mathrm{~mm}$ de espesor; seis losas con tres dosificaciones diferentes de fibra de acero $\left(5,9\right.$ y $18 \mathrm{~kg} / \mathrm{m}^{3}$, dos losas para cada dosificación), y dos losas de concreto simple. Para caracterizar las propiedades mecánicas del concreto se realizaron ensayos en compresión, módulo de elasticidad, tensión indirecta y flexión, utilizando 36 especímenes en forma de cilindros y 12 especímenes en forma de vigas. El artículo incluye la discusión no solo de las correlaciones entre las propiedades mecánicas y los parámetros de diseño del concreto reforzado con fibras de acero, sino también de las curvas carga-deflexión y las curvas tenacidad-deflexión de las losas de concreto reforzadas con las diferentes dosificaciones de fibra de acero. Los resultados demostraron que, a diferencia de la carga máxima, las deflexiones en las cargas máxima y última, y la tenacidad en la falla de las losas aumentaron significativamente a medida que se incrementó la dosificación de fibras de acero.

\section{Descriptores:}

- losas sobre terreno

- fibras de acero

- contenido de fibras

- ensayo a flexión

- tenacidad 


\begin{abstract}
Steel fiber reinforced concrete (SFRC) has become a solution to improve the concrete performance and to optimize the construction systems. The paper shows and discusses the results of an experimental program aimed at studying the flexural performance of concrete slabs-on-ground reinforced with steel fibers to be used in housing. The experimental program included flexural tests of eight 600-mm square concrete slabs having thickness of $100 \mathrm{~mm}$; six slabs with three different contents of steel fibers (5, 9 and $18 \mathrm{~kg} / \mathrm{m}^{3}$, two slabs for each fiber content), and two slabs of plain concrete. Compressive, elasticity modulus, splitting tensile and flexural test of 36 standard cylinders and 12 standard beam specimens were carried out to characterize the mechanical properties of concrete. The paper includes the discussion of not only the correlations between mechanical properties and design parameters of steel fiber reinforced concrete, but also the load-and toughness-deflection curves of concrete slabs reinforced with different contents of steel fibers. Results demonstrated that, except of peak strength, deflections at peak and ultimate load, and toughness at failure of slabs increased significantly as the fiber content augmented.
\end{abstract}

\section{Introducción}

Las losas sobre terreno se encargan de transmitir las cargas de tránsito de viviendas, parqueaderos para automóviles y bicicletas, andenes y pisos peatonales hacia el terreno. El refuerzo de estas losas se dispone para evitar que los movimientos del suelo, tales como asentamiento o expansión, generen grietas en la superficie de la losa. Como se indica en la sección E.2.2.5 de la NSR-10, la losa sobre terreno no debe conectarse estructuralmente con la estructura de cimentación y en ningún caso se debe considerar como parte integral de la cimentación. Para la construcción de losas sobre terreno es importante buscar un mejor comportamiento del concreto en términos de desempeño mecánico y durabilidad. Varias investigaciones han demostrado la capacidad de las fibras para mejorar las propiedades físicas y la durabilidad del concreto (ACI 544.5R, 2010). La adición de fibras de acero en el concreto genera un material más homogéneo con resistencia a tensión más elevada, retracción más controlada, alta resistencia al impacto (NTC-5721, 2009) y con comportamientos mecánicos caracterizados por presentar mayor número de fisuras con menores valores de abertura de fisura, que es un factor importante en los requerimientos de durabilidad (Marmol, 2010). Adicionalmente, en concretos sin fisuras se ha comprobado que la corrosión de las fibras se limita a la superficie del concreto. Luego de la corrosión de la superficie, el efecto de la corrosión no se propaga más de $2 \mathrm{~mm}$ a partir de la superficie. Las fibras de acero muestran una buena resistencia a la corrosión en elementos no fisurados, aun cuando los elementos se encuentren expuestos a agua salada (ACI 544.1R, 1996).
Varios estudios experimentales analizaron el comportamiento de losas de CRFA. Por ejemplo, Khalloo y Afshari (2005) demostraron que las losas con concreto simple fallaron repentinamente y se agrietaron sin alguna deflexión apreciable, mientras que las losas con CRFA se agrietaron gradualmente y posteriormente fallaron. Adicionalmente, Khalloo y Afshari (2005) observaron que en las losas con CRFA, la fuerza última a flexión no incrementa significativamente cuando las fibras se agregan al concreto, pero la capacidad de absorción de energía mejora significativamente. De modo similar, Sorelli et al. (2004) observaron que al aumentar el contenido de fibra, la capacidad de carga no aumenta significativamente, pero se obtiene un comportamiento más estable (poca degradación). Hadi (2008) observó que en las losas de CRFA, la deflexión última aumenta, el colapso tarda más que en la losa de concreto simple y, por tanto, la absorción de energía del espécimen de CRFA es mejor.

En este artículo se presentan y discuten los resultados de un programa experimental para estudiar el desempeño de losas de concreto sobre terreno reforzadas con fibras de acero y con cargas menores a 500 $\mathrm{kg} / \mathrm{m}^{2}$. El programa experimental incluyó ensayos a flexión de ocho losas cuadradas de concreto de 600 $\mathrm{mm}$ de lado y $100 \mathrm{~mm}$ de espesor; seis losas con tres dosificaciones de fibra de acero $\left(5,9\right.$ y $18 \mathrm{~kg} / \mathrm{m}^{3}$, dos losas para cada dosificación) y dos losas de concreto simple. Para caracterizar las propiedades mecánicas del concreto se realizaron ensayos en compresión, módulo de elasticidad, tensión indirecta y flexión, utilizando 36 especímenes en forma de cilindros y 12 especímenes en forma de vigas. Inicialmente se presentan correlaciones de los parámetros que caracteri- 
zan las propiedades mecánicas del concreto reforzado con fibras de acero. Finalmente se comparan y discuten las curvas carga-deflexión y las curvas tenacidaddeflexión de las losas de concreto simple con las losas de concreto reforzadas con diferentes dosificaciones de fibra de acero.

\section{Concreto reforzado con fibras de acero, CRFA}

Las fibras de acero se caracterizan por la relación de esbeltez y el esfuerzo de tensión del acero. La relación de esbeltez o de aspecto se define como el cociente entre longitud y diámetro de la fibra, $l_{f} / d_{f}$. De acuerdo con la sección C.3.5.8 de la NSR-10, para utilizar fibras de acero como refuerzo mínimo a cortante en vigas, estas deben tener una relación de aspecto no menor a 50 y no mayor a 100. Por otro lado, la resistencia a tensión depende de la calidad del acero, el cual puede variar entre $400 \mathrm{MPa}$ para contenido bajo y $1500 \mathrm{MPa}$ para contenido medio de carbono. Al incrementar el contenido de carbono se pueden alcanzar resistencias a tensión cercanas a $2000 \mathrm{MPa}$; este tipo de fibras son adecuadas para concretos de alta resistencia (Marmol, 2010). La dosificación, $D_{f}$ representa la masa de fibras por unidad de volumen. La fracción de volumen de fibras de acero, $V_{f}$, se utiliza para expresar la dosificación de forma adimensional y se calcula como el cociente entre $D_{f,}$ y la densidad del acero de las fibras, $\gamma_{s}$; es decir, $7850 \mathrm{~kg} / \mathrm{m}^{3}$. El parámetro $V_{f}$ se expresa usualmente en porcentaje.

\section{Características mecánicas del CRFA}

La adición de fibras al concreto no genera un efecto importante en la resistencia del concreto a compresión; solo suelen producirse ligeros incrementos y decrementos de la resistencia (Yazici, 2007). En los concretos jóvenes (antes de los 28 días), la carga máxima de los concretos con fibra aumenta, pero a medida que el material madura, este aumento se reduce y luego la capacidad de absorción de energía y la ductilidad se concentran en la región post-fisura (Marmol, 2010). La resistencia por compresión diametral y su deformación unitaria se mantienen casi constantes para el concreto simple (CS) y el CRFA, ya que a partir de este punto se inicia la contribución de las fibras de acero. Sin embargo, la resistencia máxima por compresión diametral y su deformación unitaria incrementan con la adición de fibras, ya que las fibras en este punto proporcionan un efecto de "enganche" en el concreto, lo que genera un incremento en sus capacidades de resistencia y desplazamiento (Carrillo et al., 2014).
Por la dificultad que implica realizar el ensayo de tensión directa en especímenes de concreto, este método no es muy empleado para evaluar la resistencia a tensión del CRFA. El ensayo de flexo-tensión, además de ser más simple que el de tensión directa, simula las condiciones de carga para muchas aplicaciones prácticas. La prueba de flexión se considera una de las más representativas para el CRFA, ya que a partir de ella se puede estimar la capacidad de deformación que la fibra le proporciona al concreto luego de alcanzar el concreto inicial por flexión (Carrillo et al., 2012). Al adicionar fibras de acero al concreto, el incremento de la resistencia a flexión es considerablemente mayor que el de la resistencia a compresión y tensión diametral. Esto se debe al comportamiento dúctil del CRFA en la zona fisurada por tensión; es decir, el desarrollo de resistencias residuales (Hannant, 1978).

En el ensayo a flexión normalmente se determina la resistencia a la primera fisura, la resistencia máxima y resistencias residuales a flexo-tensión. El incremento de la resistencia a primera fisura debido a la adición de fibras de acero es mínimo, lo cual indica que esta propiedad depende básicamente de la matriz y muy poco del contenido de fibras, del tamaño y de la forma de estas. Por tanto, para la resistencia a primera fisura resulta más rentable mejorar las características de la matriz con el uso de aditivos, humo de sílice, entre otros (ACHE, 2000). La resistencia máxima depende principalmente del volumen de fibras y de la esbeltez de estas; por ejemplo, se pueden lograr incrementos de hasta $100 \%$ respecto a la resistencia de la matriz. El empleo de mayores volúmenes de fibras y fibras de mayores longitudes origina incrementos de hasta $150 \%$ en la resistencia máxima a flexo-tensión. Con contenido de bajas dosificaciones y fibras de esbeltez baja también se logran incrementos significativos de resistencia (Hannant, 1978).

\section{Programa experimental}

El programa experimental incluyó ensayos a flexión de ocho losas cuadradas de concreto de $600 \mathrm{~mm}$ de lado y $100 \mathrm{~mm}$ de espesor; seis losas con diferentes dosificaciones de fibra de acero $\left(5,9\right.$ y $18 \mathrm{~kg} / \mathrm{m}^{3}$, dos losas para cada dosificación), y dos losas de concreto simple (CS). Los ensayos de flexión de losas se planearon para estudiar el desempeño de las losas por medio de las curvas carga-deflexión. Para caracterizar las propiedades mecánicas del concreto se realizaron ensayos en compresión, módulo de elasticidad, tensión indirecta y flexión, utilizando 36 especímenes en forma de cilindros y 12 especímenes en forma de vigas. 


\section{Tipo de concreto}

El concreto que se utilizó para la investigación fue de peso normal con resistencia nominal a compresión de $21 \mathrm{MPa}$, tamaño máximo de agregado de $12.7 \mathrm{~mm}(1 / 2$ pulg.) y asentamiento de $254 \mathrm{~mm}$ (10 pulg.). La composición del concreto por $\mathrm{m}^{3}$ fue de $283 \mathrm{~kg}$ (90.7 L) de cemento Tipo III, $71 \mathrm{~kg}$ (33.6 L) de ceniza proveniente de la Termoeléctrica de Paipa (Boyacá, Colombia), $850 \mathrm{~kg}$ (330.7 L) de arena, $896 \mathrm{~kg}$ (336.8 L) de grava, $219 \mathrm{~kg}$ (219 L) de agua y $2 \mathrm{~kg}$ (1.7 L) de aditivo (ViscoCrete $2100 \mathrm{y}$ Plastiment AD-20). El concreto se premezcló y suministró por la empresa Argos.

Para determinar las propiedades del concreto en estado fresco se realizaron pruebas de asentamiento, masa unitaria y contenido de aire. Para caracterizar las propiedades mecánicas del concreto se realizaron ensayos de compresión, módulo de elasticidad, tensión indirecta (compresión diametral) y flexión. En general, el proyecto incluyó el ensayo de 36 especímenes de forma cilíndrica con $150 \mathrm{~mm}$ de diámetro y $300 \mathrm{~mm}$ de longitud, y 12 vigas de sección cuadrada con $150 \mathrm{~mm}$ de lado, y $600 \mathrm{~mm}$ de longitud (3 especímenes para cada tipo de losa y para cada tipo de ensayo).

\section{Tipo y dosificación de fibras de acero}

Las dosificaciones de fibra que se emplearon 5, 9 y 18 $\mathrm{kg} / \mathrm{m}^{3}$ ( $\mathrm{m}^{3}$ de concreto), las cuales equivalen a fracciones de volumen de $0.06 \%, 0.11 \%$ y $0.23 \%$. Las fibras de acero son de referencia RL-45/50-BN (Dramix malla en bolsa), con gancho en serie 3D, longitud $l_{f}$ de $50 \mathrm{~mm}$, diámetro $d_{f}$ de $1.05 \mathrm{~mm}$, relación de aspecto $l_{f} / d_{f}$ de $48 \mathrm{y}$ resistencia a tensión de $1115 \mathrm{MPa}$.

Las fibras se mezclaron en sitio dentro de la mezcladora del camión de concreto. Para garantizar una distribución uniforme de las fibras de acero en la matriz del concreto y prevenir segregación o agrupación de las mismas, el mezclado de las fibras se realizó según indicaciones del fabricante y, por tanto, la velocidad de adición de fibras fue de $1.7 \mathrm{~kg} / \mathrm{min}$ y el tiempo de mezclado fue de 5 minutos. De modo similar al estudio de Carrillo et al. (2014), para agregar las fibras de una determinada dosificación se calculó el volumen presente de concreto en la mezcladora, es decir, se consideró el volumen extraído de concreto para elaborar los especímenes de las dosificaciones precedentes. Cuando finalizó la elaboración de especímenes de una determinada dosificación, se adicionó al concreto la fibra necesaria para lograr la dosificación que se necesitaba.

El ensayo de contenido de fibras se llevó a cabo según los lineamientos de la norma EN-14488-7 (2007).
Para tomar muestras de CRFA en estado fresco se utilizaron tres cilindros de $150 \mathrm{~mm}$ de diámetro y $300 \mathrm{~mm}$ de altura. Para inactivar el material cementante, se adicionó suficiente agua a las mezclas. Luego se secaron las fibras a temperatura ambiente y se extrajeron las fibras con un imán. Finalmente se pesaron las fibras para obtener el valor real en cada dosificación, a partir del peso y el volumen de concreto en los cilindros.

\section{Elaboración y curado de especímenes}

Las losas se elaboraron en moldes metálicos. Para prevenir el alineamiento preferencial de las fibras de acero dentro del concreto, el método de vibración de la matriz de CRFA fue diferente al del concreto simple. Para las losas de concreto simple (CS) se agregó una sola capa de concreto y se aplicaron 100 apisonadas con una barra de acero recta y con extremos redondeados. Para las losas de CRFA se vertió una sola capa de concreto y se aplicaron 100 golpes externos con un mazo de cabeza de goma. Para la elaboración de los cilindros de CS se agregaron tres capas iguales en los moldes metálicos, cada capa se apisonó con 25 golpes utilizando la barra de acero. En los cilindros de CRFA, a cada capa se le aplicaron 25 golpes externos con el mazo de cabeza de goma. Para la fabricación de las vigas de CS se agregaron dos capas iguales, cada capa se apisonó con 64 golpes utilizando la barra de acero. Para la elaboración de las vigas de CRFA se aplicaron 64 golpes externos en cada capa con un mazo de cabeza de goma.

El desencofrado de las probetas se realizó a las $24 \pm 8$ horas. El curado de los especímenes se realizó inmediatamente después de desmoldar los especímenes. El curado consistió en la aplicación, sobre cada superficie de los especímenes, de la membrana de curado Antisol Blanco de Sika (2015). La membrana se aplicó con brocha a dos capas.

\section{Configuración de ensayo de losas}

El ensayo de absorción de energía se realizó de acuerdo con los lineamientos de la norma NTC-5721 (2009). Los ensayos se realizaron en un marco de carga con capacidad de $100 \mathrm{kN}$, que cuenta con una rótula para garantizar la carga de forma axial. El marco dispone de un marco metálico cuadrado rígido y nivelado, con medidas internas de $500 \mathrm{~mm}$ de lado, con un borde perimetral sobre el cual se apoya el espécimen. El elemento sobre el cual se aplica la carga es una platina cuadrada de acero de $100 \mathrm{~mm}$ de lado y $20 \mathrm{~mm}$ de espesor (figura 1a). La velocidad de aplicación de la carga en el punto central de la losa fue de $1.5 \mathrm{~mm} / \mathrm{min}$. Para medir las 
deflexiones se utilizaron cinco transductores de desplazamiento lineal; cuatro de ellos estuvieron montados en un dispositivo especial patentado (Carrillo et al., 2015) que se sujeta en los extremos de la parte superior de la probeta. Estos cuatro transductores de desplazamiento son de marca TML, referencia CDP-10, con capacidad máxima de $10 \mathrm{~mm}$. Adicionalmente se colocó un transductor de desplazamiento en el centro de la probeta. Este transductor es de marca Controls, referencia 82P0331/D1, con capacidad máxima de 10mm. En los ensayos de flexión de losas se determinaron parámetros de desempeño tales como carga máxima $\left(P_{\max }\right)$, carga última asociada a la falla de la losa $\left(P_{u}\right)$, deflexión en $P_{u}\left(\delta_{u}\right)$ y tenacidad en flexión de losas $\left(T_{l}\right)$.

\section{Configuración de ensayos mecánicos del concreto}

Los ensayos de resistencia a compresión y módulo de elasticidad/relación de Poisson del concreto se realizaron conforme a los lineamientos de la normas NTC-673 (2000) y NTC-4025 (2006), respectivamente. Los ensayos se llevaron a cabo en una maquina universal con capacidad máxima de 2000 kN en compresión. Para garantizar que la carga se distribuyera uniformemente, se utilizó neopreno en los extremos de los cilindros. Para medir las deformaciones transversales y longitudinales y así determinar el módulo de elasticidad y la relación de Poisson, al espécimen se le acopló el dispositivo compresómetro-extensómetro que se muestra en la figura 1b. Adicionalmente, al espécimen se le adhirió un deformímetro capaz de medir la deformación transver-

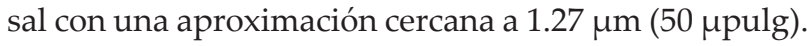
En este estudio, el módulo de elasticidad del concreto es el valor que representa la pendiente de la línea que une los puntos esfuerzo-deformación longitudinal correspondiente a una deformación de 0.00005 y 40\% de la carga máxima obtenida de los ensayos de resistencia a compresión. Por tanto, el módulo representa el módulo secante a $40 \%$ de la resistencia máxima. La relación de Poisson corresponde al valor de la pendiente de la línea que une los puntos esfuerzo-deformación transversal a una deformación de 0.00005 y $40 \%$ de la carga máxima obtenida de los ensayos de resistencia a compresión.

Los ensayos de tensión por compresión diametral se realizaron siguiendo los lineamientos de la norma NTC-722 (2000). El dispositivo de ensayo (figura 1c) consiste en dos guías para garantizar que la carga se aplique en el centro del diámetro del cilindro. La carga se aplicó con la misma máquina utilizada para los ensayos a compresión. Aunque la norma NTC-722 (2000) no indica la medición de desplazamientos, en este estudio se midió el desplazamiento transversal para trazar la curva completa esfuerzo-deformación y así observar la capacidad de deformación luego del primer pico de resistencia a tensión por compresión diametral $\left(f_{o}\right)$. El desplazamiento transversal se midió con dos transductores marca TML, referencia CDP-5, con capacidad máxima de $5 \mathrm{~mm}$. Los transductores se ubicaron de forma paralela al eje transversal y estuvieron centrados alrededor de la mitad de la altura del diámetro del espécimen en cada una de sus dos caras. La deformación transversal en cada cara se calculó como el cociente entre las lecturas de desplazamiento y la separación de los dos anclajes para sujetar cada transductor. Luego se calculó la deformación promedio a partir de las deformaciones en cada cara. En la figura 2a se indican los principales parámetros asociados a la curva envolvente medida durante el ensayo de tensión por compresión diametral, donde

$f_{o}=$ resistencia a tensión por compresión diametral

$f_{t}=$ resistencia máxima

$f_{p}=$ resistencia asociada al inicio de la contribución de las fibras

$f_{u t}=$ resistencia última a tensión por compresión diametral

$\varepsilon_{o^{\prime}} \varepsilon_{t^{\prime}} \varepsilon_{p}$ y $\varepsilon_{u t}=$ deformaciones unitarias asociadas a $f_{o^{\prime}} f_{t^{\prime}}$, $f_{p} \mathrm{y} f_{u t}$, respectivamente

$T_{t}=$ tenacidad en compresión diametral
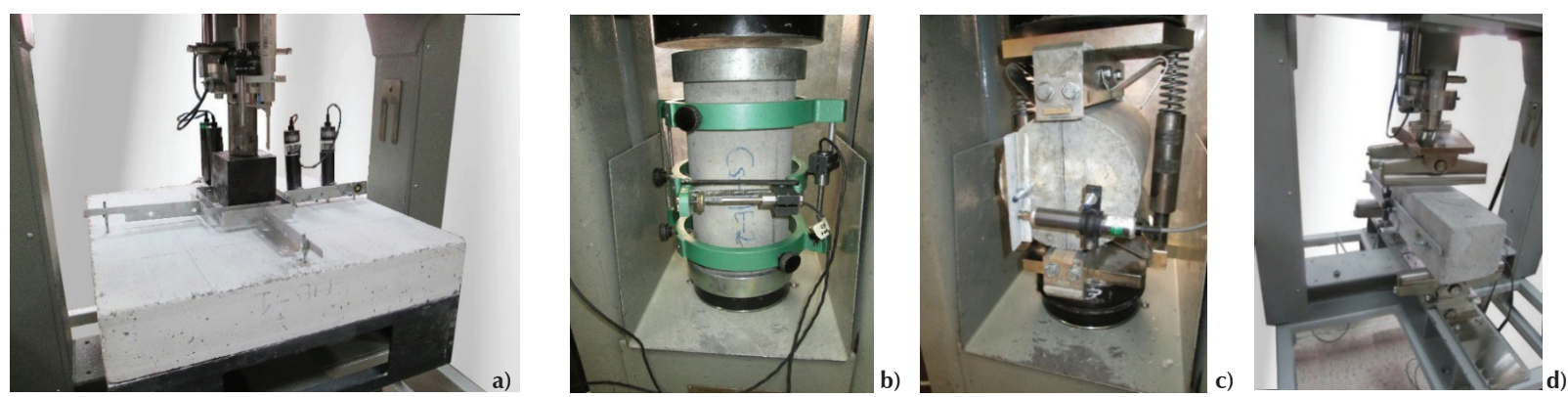

Figura 1. Configuración de ensayos: a) flexión de losas, b) módulo de elasticidad, c) tensión indirecta, d) flexión 
Los ensayos a flexión de vigas se realizaron con base en los lineamientos de la NTC-2871 (2004) para vigas de concreto sin fibras y con base en la ASTM-1609 (2012) para vigas de concreto reforzadas con fibras de acero. El ensayo se realizó en el mismo marco de carga utilizado en el ensayo de losas, que también es capaz de aplicar la carga en el tercio medio de la muestra sin excentricidad. Los rodillos de soporte son libres de rotar sobre sus ejes y la distancia entre apoyos debe ser de $450 \mathrm{~mm}$. Para las vigas con concreto simple se utilizó una velocidad de 129.4 N/s, aproximadamente. Para registrar mayor número de datos en la rama después de la primera fisura de los elementos reforzados con fibra de acero, se utilizó una velocidad de carga de $30 \mathrm{~N} / \mathrm{s}$. Para medir la deflexión en el centro de la luz se utilizaron dos transductores de desplazamiento digitales marca TML, referencia CDP-5, con capacidad de $5 \mathrm{~mm}$. Estos transductores se ubicaron en la mitad de la distancia entre apoyos, uno a cada lado, con soportes apropiados unidos a la muestra (figura 1d). El promedio de las dos mediciones representa la deflexión neta. La tenacidad en flexión, $T_{f}$, se obtuvo a partir del área bajo la curva carga-deflexión de la viga, tal como se establece en la norma ASTM-C-1609 (2012).

En la figura $2 b$ se muestra la curva característica de vigas de concreto a flexión. Los puntos principales son la carga de primer pico, $P_{p}$, la deflexión en la carga $P_{p}$ $\delta_{p}$ las resistencias residuales asociadas a deflexiones al centro del claro equivalentes a $l_{c} / 300$ y $l_{c} / 150$, y la tenacidad en flexión $T_{f}$. Según el criterio de la sección C5.6.6.2 de la NSR-10, se considera aceptable el CRFA para resistir fuerza cortante, cuando la resistencia residual equivalente a $l_{c} / 300$ sea igual o mayor de $90 \%$ y la resistencia residual equivalente a $l_{c} / 150$ sea igual o mayor de $75 \%$ de la resistencia del primer pico medida en el ensayo realizado conforme los lineamientos de la norma ASTM-1609 (2012). En este estudio también se determinaron la resistencia última $\left(f_{u}\right)$ y deflexión última $\left(\delta_{u}\right)$, que corresponden al instante en que se presenta la falla del espécimen.
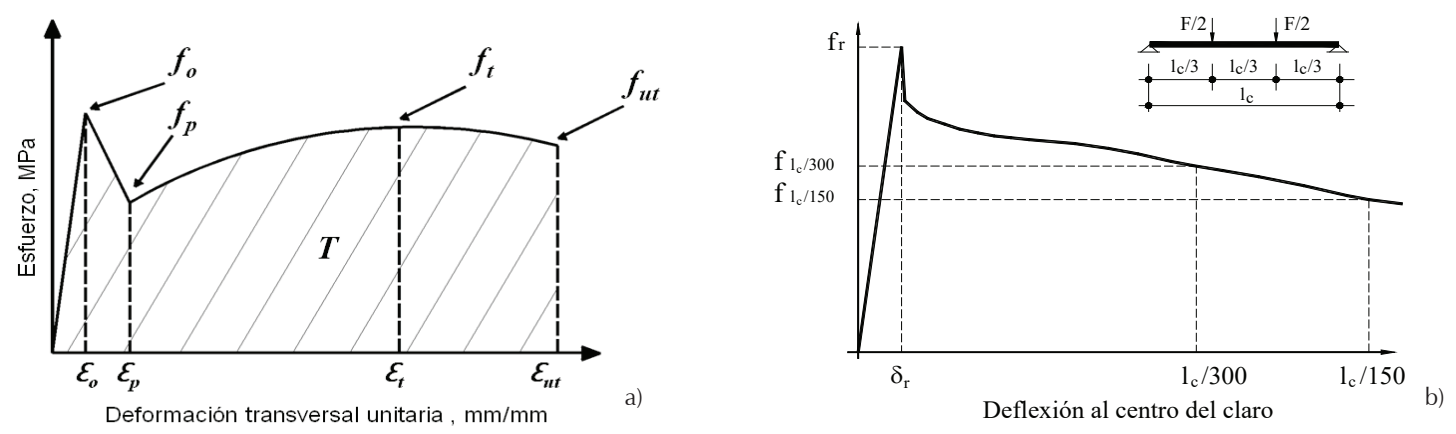

Figura 2. Curvas características: a) tensión indirecta, b) flexión en vigas dos experimentalmente.

\section{Propiedades de los materiales}

\section{Contenido de fibras}

En la tabla 1 se muestran los valores de dosificación real
(valores medidos), los cuales fueron claves para el aná-
lisis de la información experimental de los especíme-
nes. Para propósitos del análisis de resultados, a partir
de esta sección se utilizarán los valores reales (medi-
dos) de la dosificación de fibras de acero.

En la tabla 1 se muestran los valores de dosificación real
(valores medidos), los cuales fueron claves para el aná-
lisis de la información experimental de los especíme-
nes. Para propósitos del análisis de resultados, a partir
de esta sección se utilizarán los valores reales (medi-
dos) de la dosificación de fibras de acero.

En la tabla 1 se muestran los valores de dosificación real
(valores medidos), los cuales fueron claves para el aná-
lisis de la información experimental de los especíme-
nes. Para propósitos del análisis de resultados, a partir
de esta sección se utilizarán los valores reales (medi-
dos) de la dosificación de fibras de acero.

En la tabla 1 se muestran los valores de dosificación real
(valores medidos), los cuales fueron claves para el aná-
lisis de la información experimental de los especíme-
nes. Para propósitos del análisis de resultados, a partir
de esta sección se utilizarán los valores reales (medi-
dos) de la dosificación de fibras de acero.

En la tabla 1 se muestran los valores de dosificación real
(valores medidos), los cuales fueron claves para el aná-
lisis de la información experimental de los especíme-
nes. Para propósitos del análisis de resultados, a partir
de esta sección se utilizarán los valores reales (medi-
dos) de la dosificación de fibras de acero.

En esta sección se presentan y se discuten los resultados experimentales del estudio. Inicialmente se presentan las propiedades del concreto en estado fresco y las propiedades mecánicas del concreto en estado endurecido. Adicionalmente, se muestran correlaciones de los parámetros que caracterizan las propiedades mecánicas del concreto reforzado con fibras de acero. Luego se presentan las curvas carga-deflexión y las curvas tenacidad-deflexión de las losas con las diferentes dosificaciones de fibra de acero. Para estudiar los datos medidos se utilizaron parámetros estadísticos tales como el promedio $(X)$, desviación estándar $(S)$, coeficiente de variación $(\mathrm{CV})$ y coeficiente de correlación $(r)$. El coeficiente de variación $(\mathrm{CV})$ es directamente proporcional a la dispersión y representa la relación porcentual entre la desviación estándar $(S)$ y el promedio $(X)$. El coeficiente $r$ mide la intensidad de la relación lineal entre los valores calculados por una ecuación y los datos obteni-

En esta sección se presentan y discuten los resultados del contenido real de fibras, las propiedades del concreto en estado fresco, tales como asentamiento, masa unitaria y contenido de aire, los parámetros de las curvas esfuerzo deformación-deformación en compresión axial y en tensión por compresión diametral, así como los parámetros de la curva carga-deflexión en flexión. 
Tabla 1. Propiedades mecánicas del concreto en compresión

\begin{tabular}{|c|c|c|c|c|c|}
\hline Edad, días & & 188 & 188 & 188 & 188 \\
\hline$D_{f-\text { nominal }}, \mathrm{kg} / \mathrm{m}^{3}$ & & 0 & 5.0 & 9.0 & 18.0 \\
\hline \multirow{2}{*}{$D_{f,} \mathrm{~kg} / \mathrm{m}^{3}$} & $X$ & 0 & 3.4 & 7.6 & 14.1 \\
\hline & $C V, \%$ & 0 & 7.9 & 6.3 & 1.5 \\
\hline$V_{f,} \%$ & & 0 & 0.04 & 0.10 & 0.18 \\
\hline $\mathrm{V}_{\mathrm{f}, \mathrm{f}} \times\left(\mathrm{l}_{\mathrm{f}} / \mathrm{d}_{\mathrm{f}}\right)$ & & 0.00 & 1.92 & 4.80 & 8.64 \\
\hline \multirow[t]{2}{*}{$f_{c}, \mathrm{MPa}$} & $X$ & 28.7 & 30.2 & 31.2 & 31.7 \\
\hline & $C V, \%$ & 3.3 & 3.6 & 3.5 & 1.9 \\
\hline \multirow{2}{*}{$\varepsilon_{0}\left(\right.$ deformación asociada a $\left.f_{c}\right)$} & $X$ & 0.0018 & 0.0023 & 0.0025 & 0.0028 \\
\hline & $C V, \%$ & 5.5 & 27.7 & 15.8 & 26.7 \\
\hline \multirow{2}{*}{$\begin{array}{l}E_{c}, \mathrm{MPa} \\
\text { (compresómetro) }\end{array}$} & $X$ & 17606 & 18034 & 17250 & 17600 \\
\hline & $C V, \%$ & 3.9 & 4.6 & 2.3 & 0.2 \\
\hline \multirow{2}{*}{$\begin{array}{l}E_{c}, \mathrm{MPa} \\
\text { (deformímetro) }\end{array}$} & $X$ & 17601 & 20931 & 19175 & 18057 \\
\hline & $C V, \%$ & 8.7 & 6.1 & 0.3 & 0.1 \\
\hline$E_{c(\text { deformimetro) }} / E_{c(\text { compresometro })}$ & - & 1.00 & 1.16 & 1.11 & 1.03 \\
\hline \multirow[b]{2}{*}{$v$} & $X$ & 0.16 & 0.18 & 0.16 & 0.16 \\
\hline & $C V, \%$ & 3.5 & 6.2 & 3.5 & 8.6 \\
\hline
\end{tabular}
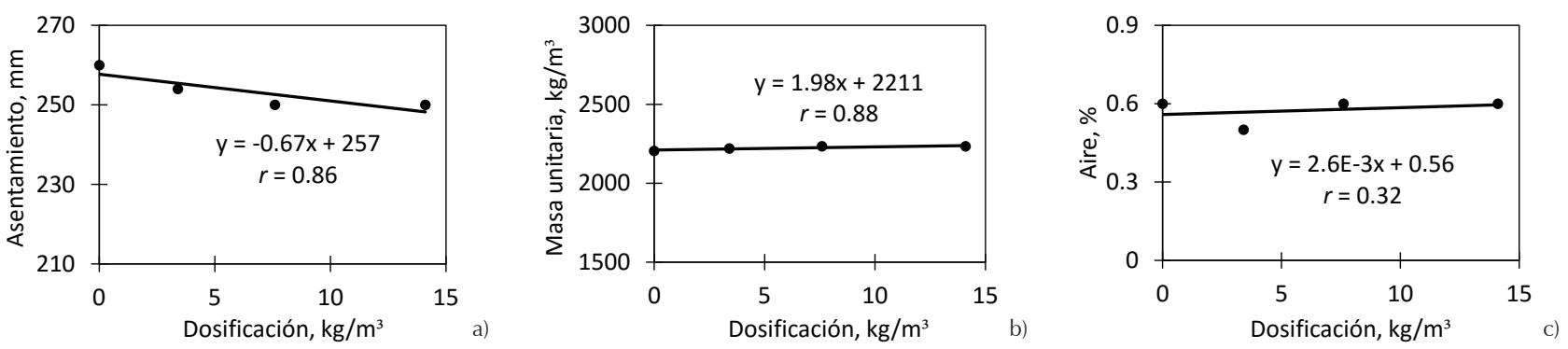

Figura 3. Propiedades del concreto en estado fresco: a) asentamiento, b) masa unitaria, c) contenido de aire

Asentamiento, masa unitaria y contenido de aire

Como se observa en la figura $3 \mathrm{a}$, el asentamiento disminuyó levemente cuando se aumentó el contenido de fibras. Esta disminución se debe al efecto de "enganche" de las fibras en el concreto. En la figura $3 b$ se observa que el intervalo de los valores medidos de la masa unitaria del concreto $\left(2205 \mathrm{~kg} / \mathrm{m}^{3}\right.$ y $\left.2234 \mathrm{~kg} / \mathrm{m}^{3}\right)$ se encuentran en el límite establecido por la NSR-10 para concreto simple de peso normal $\left(2155 \mathrm{~kg} / \mathrm{m}^{3}\right.$ y $\left.2550 \mathrm{~kg} / \mathrm{m}^{3}\right)$. El contenido de aire no registró cambios significativos a medida que se aumentó la dosificación de fibras (figura $3 c)$. Esto se debe a que la pequeña diferencia entre las dosificaciones empleadas no incentivó cambios significativos de esta propiedad.

\section{Comportamiento en compresión}

El valor promedio de la resistencia a compresión a edad de 60 días fue de 27.0 MPa. Este valor es 28.5\% (27/21) mayor que el valor de la resistencia nominal a compresión del concreto (21 MPa). Los ensayos de las losas de concreto se realizaron a una edad cercana a los 190 días, donde el concreto simple presentó una resistencia a compresión de 28.7 MPa; es decir, se obtuvo un aumento de $6.3 \%$ respecto a la resistencia a los 60 días. En la figura 4 se muestran algunas de las curvas esfuerzodeformación de los concretos utilizados.

En la tabla 1 se presenta, para cada dosificación, las propiedades mecánicas promedio del CRFA sometidos a esfuerzos de compresión. En la tabla se muestran los valores del módulo de elasticidad obtenidos a partir de los registros de desplazamiento en el compresómetro y los registros de los deformímetros. Como se muestra en la tabla, los valores obtenidos con los deformímetros en promedio son $7.4 \%$ mayores a los obtenidos con el compresómetro. Por tanto, los valores de módulo de elasticidad reportados en este estudio corresponden a los obtenidos a partir de las lecturas del compresómetro, ya que fueron los valores más conservadores; es decir, 

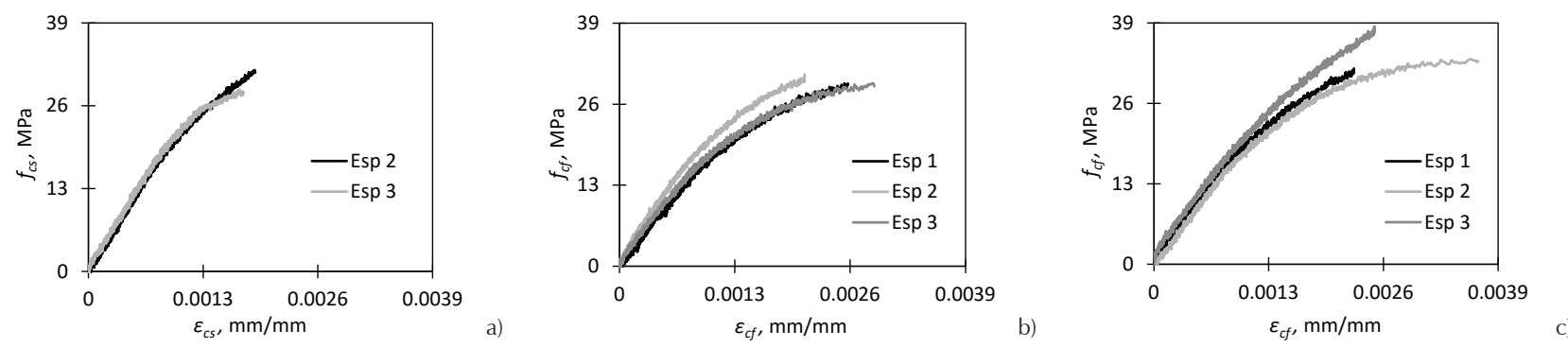

Figura 4. Curvas esfuerzo-deformación del concreto en compresión: a) CS, b) $D_{f}=3.4 \mathrm{~kg} / \mathrm{m}^{3}$, c) $D_{f}=14.1 \mathrm{~kg} / \mathrm{m}^{3}$
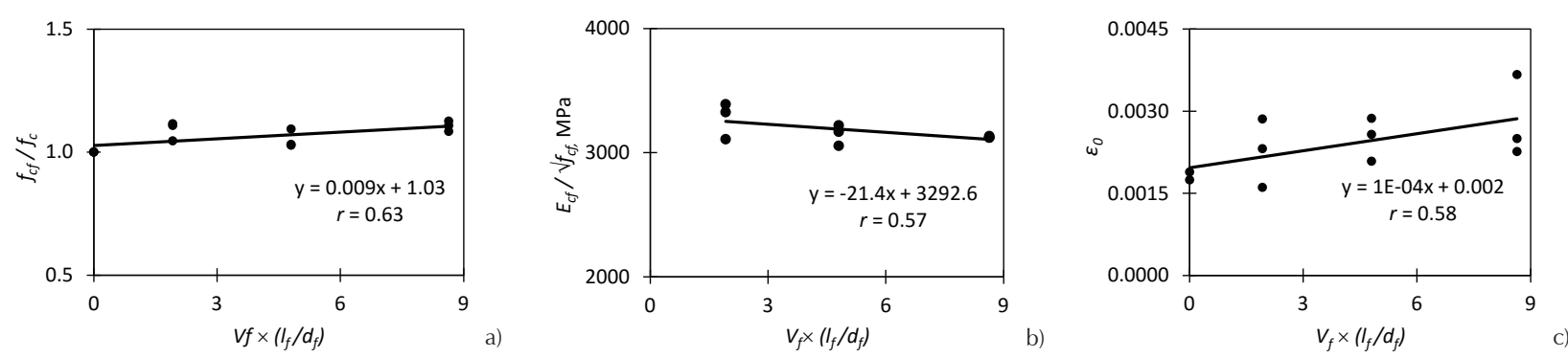

Figura 5. Tendencias de las propiedades mecánicas del concreto en compresión: a) resistencia a compresión, b) módulo de elasticidad, c) deformación asociada a $f_{c}\left(\varepsilon_{0}\right)$

valores menores a los obtenidos con las lecturas de los deformímetros.

En la figura 5 se muestra la variación de las propiedades mecánicas en compresión con el producto $V_{f} \times\left(l_{f} / d_{f}\right)$. Para el caso de la resistencia a compresión, en la figura 5 a se muestra el cociente entre la resistencia a compresión del CRFA y la del concreto simple $\left(f_{c f} / f_{c}\right)$. Para el módulo de elasticidad, en la figura $5 \mathrm{~b}$ se muestra el cociente entre $E_{c f}$ y la raíz cuadrada de $f_{c f}\left(E_{c f} / \sqrt{ } f_{c f}\right)$.

En la figura 5 a se observa que la resistencia a compresión aumentó levemente a medida que incrementó el producto $V_{f} \times\left(l_{f} / d_{f}\right)$. El ligero aumento de la resistencia a compresión se debe a que se utilizaron dosificaciones bajas que no permitieron identificar el cambio esperado; por ejemplo, para dosificaciones mayores a $15 \mathrm{~kg} / \mathrm{m}^{3}$, Gallo et al. (2013) observaron que la resistencia a compresión del concreto tiende a disminuir cuando se agregan fibras de acero. Teniendo en cuenta que la línea de tendencia tiene pendiente poco pronunciada, el coeficiente de correlación es bajo $(r=0.63)$. En la figura $5 \mathrm{~b}$ se muestra que el módulo de elasticidad disminuye de acuerdo con el incremento del producto $V_{f} \times\left(l_{f} / d_{f}\right)$, debido a una disminución en la cantidad de agregado grueso por la presencia de las fibras. Una tendencia similar de reducción se observó para el coeficiente de Poisson, la cual se debe al confinamiento que las fibras generan en la matriz, tal como se observó en el estudio de Gallo et al. (2013). Los coeficientes de correlación para las tendencias del módulo de elasticidad $(r=0.57)$ y la relación de Poisson $(r=0.54)$ son bajos, debido a que las pendientes de las curvas es baja. En la figura $5 c$ se observa que a medida que incrementa el contenido de fibra de acero, la deformación unitaria $\varepsilon_{0}$ aumenta; por ejemplo, para el concreto reforzado con la dosificación de $14.1 \mathrm{~kg} / \mathrm{m}^{3}$ (mayor dosificación utilizada en el estudio), la capacidad de deformación unitaria $\varepsilon_{0}$ respecto al concreto simple incrementó $56 \%$, en promedio (0.0028/0.0018). El incremento de la deformación $\varepsilon_{0}$ se presentó debido al efecto de "enganche" de las fibras en la matriz del concreto; es decir, mayor capacidad de deformación asociada a la resistencia.

\section{Tensión por compresión diametral}

En la figura 6 se muestran las curvas esfuerzo-deformación medidas durante los ensayos de tensión por compresión diametral para el CRFA. En la tabla 2 se muestran los valores estadísticos medidos de los principales parámetros de la curva (figura 2a). Los valores de resistencia a tensión por compresión diametral se expresaron en función de la raíz cuadrada de la resistencia a compresión del CS, $\downarrow f_{c^{\prime}}$ de forma similar al formato de ecuaciones propuestas en estudios experimentales previos tales como Carrillo et al. $(2014,2013)$.

En la figura 7 se presenta la relación entre las propiedades del CRFA sometido a esfuerzos de tensión por compresión diametral, y el producto del volumen de fibras y relación de esbeltez de las fibras $\left[V_{f} \times\left(l_{f} / d_{f}\right)\right]$. En la tabla 2 se observa que la resistencia 
a tensión por compresión diametral $\left(f_{0}\right)$ permanece casi inalterable para todas las mezclas; adicionalmente, en la figura 7a se observa que no hay un cambio significativo de la deformación $\varepsilon_{o}$ con el aumento del producto $V_{f} \times\left(l_{f} / d_{f}\right)$. Estas tendencias se deben a que estos parámetros están asociados a la formación de la primera grieta, es decir, en el punto donde no ha iniciado la contribución de fibras. En la figura $7 \mathrm{~b}$ se observa que la resistencia asociada al inicio de la contribución de las fibras incrementa levemente a medida que $V_{f} \times\left(l_{f} / d_{f}\right)$ aumenta. Esto se debe a que el descenso de la resistencia después de la primera fisura es menor, de acuerdo con el aumento en la dosificación de fibras. La disminución de la degradación también afecta la deformación $\varepsilon_{p}$ asociada a este parámetro (figura 7c), pues esta deformación disminuye al aumentar el producto $V_{f} \times\left(l_{f} / d_{f}\right)$. En las figuras 7e y $15 \mathrm{f}$ se observa que $f_{t}$ y $\varepsilon_{t}$ incrementan porque $V_{f} \times\left(l_{f} / d_{f}\right)$ aumenta, ya que en ese punto las fibras proporcionan al concreto no solo la máxima capacidad de resistencia, sino también una capacidad de deformación a tensión mayor que la que se presenta en un concreto $\sin$ fibras. En la figura $7 \mathrm{~g}$ se observa que $f_{u t}$ aumenta con el producto $V_{f} \times\left(l_{f} / d_{f}\right)$, ya que las fibras logran que la tasa de degradación disminuya. En la figura 7 h se observa una disminución de la deformación $\varepsilon_{u t}$ ya que las fibras logran restringir o mantener la capacidad de deformación máxima de la matriz de concreto. Tal como se esperaba, la tenacidad $T_{t}$ aumenta conforme aumenta la dosificación de fibras (figura 7i). Es importante destacar que, con excepción de $\varepsilon_{0}$, las tendencias de los parámetros medidos son similares a las tendencias reportadas por Carrillo et al. (2013).

\section{Tensión por flexión}

En la figura 8 se muestran las curvas carga-deflexión medidas durante los ensayos de flexión de vigas de
CRFA. En la tabla 3 se muestran los valores estadísticos medidos de los principales parámetros de la curva (figura $2 b$ ). En cuanto a esfuerzos de tensión por flexión, $f_{r}$ es resistencia al agrietamiento o módulo de ruptura, $f_{l c / 300}$ y $f_{l c / 150}$ son las resistencias residuales asociadas a deflexiones en el centro del claro equivalentes a $l_{c} / 300 \mathrm{y}$ a $l_{c} / 150$, respectivamente; y $f_{u}$ es la resistencia última; adicionalmente, $\delta_{u}$ es la deflexión última, $\mathrm{y}_{f}$ es la tenacidad en tensión por flexión en Joule (J). El punto último corresponde al punto donde colapsa el espécimen y ocurre una pérdida de resistencia súbita.

Para determinar el efecto normalizado de las fibras de acero sobre la resistencia a tensión por flexión del concreto, se obtuvieron los cocientes entre la resistencia al agrietamiento y la raíz cuadrada de la resistencia a compresión del CRFA $\left(f_{r} / f_{c}\right)$. Adicionalmente, para verificar lo indicado en la sección C5.6.6.2 de la NSR10 , se obtuvieron los cocientes entre las resistencias residuales y el módulo de ruptura del CRFA $\left(f_{l c / 300} / f_{r} \mathrm{y}\right.$ $\left.f_{l c / 150} / f_{r}\right)$. En este estudio no se observó un segundo pico de resistencia después del agrietamiento y, por tanto, no se reportan valores asociados a la resistencia máxima, $f_{\max }$. La tenacidad en tensión por flexión $\left(T_{f}\right)$ se calculó hasta la deflexión $\delta_{u}$, que se asocie a la resistencia última $\left(f_{u}\right)$.

Los ensayos de flexión de vigas demostraron que la resistencia al agrietamiento o módulo de ruptura, $f_{r}$ y la deflexión asociada a esta resistencia, $\delta_{p}$, no varían significativamente al incrementar el contenido de fibra, pues a partir de este punto de la curva inicia el aporte de las fibras. En la figura 9 se muestran las relaciones entre el producto $V_{f} \times\left(l_{f} / d_{f}\right)$ y las principales propiedades mecánicas que determinan la resistencia residual y la absorción de energía a tensión por flexión del CRFA. En la figura 9c se observa que la tenacidad $\left(T_{f}\right)$ aumentó levemente al incrementar el contenido de fibras de acero. En las figura 9a y $9 \mathrm{~b}$ se observa que las resistencias residuales asociadas a las deflexiones en el claro equivalen-
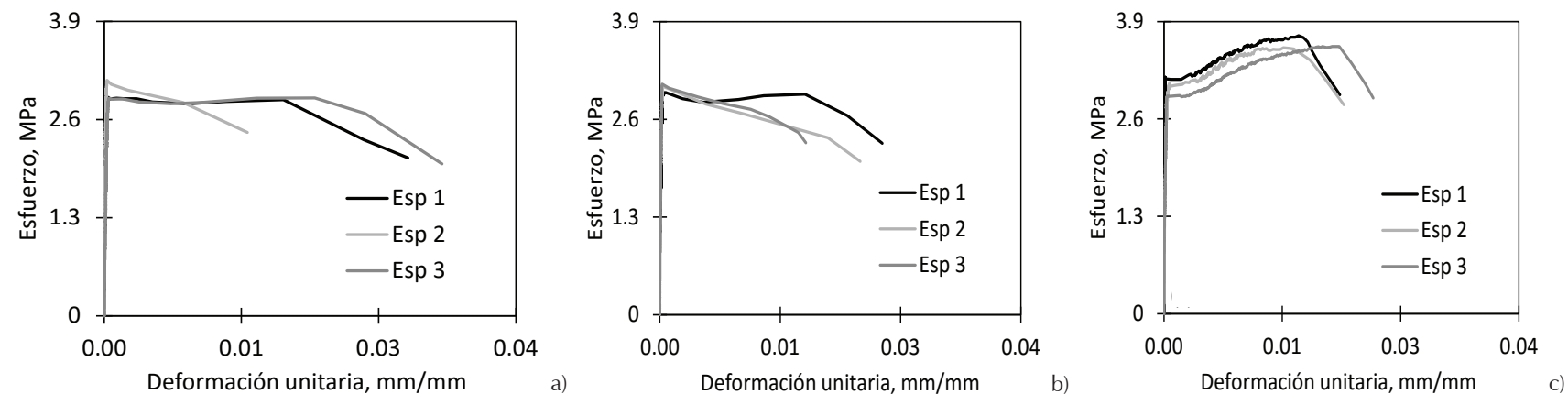

Figura 6. Curvas esfuerzo-deformación del concreto en tensión por compresión diametral: a) $\left.D_{f}=3.4 \mathrm{~kg} / \mathrm{m}^{3}, \mathrm{~b}\right) D_{f}=7.6 \mathrm{~kg} / \mathrm{m}^{3}$, C) $D_{f}=14.1 \mathrm{~kg} / \mathrm{m}^{3}$ 
Tabla 2. Propiedades mecánicas en tensión por compresión diametral

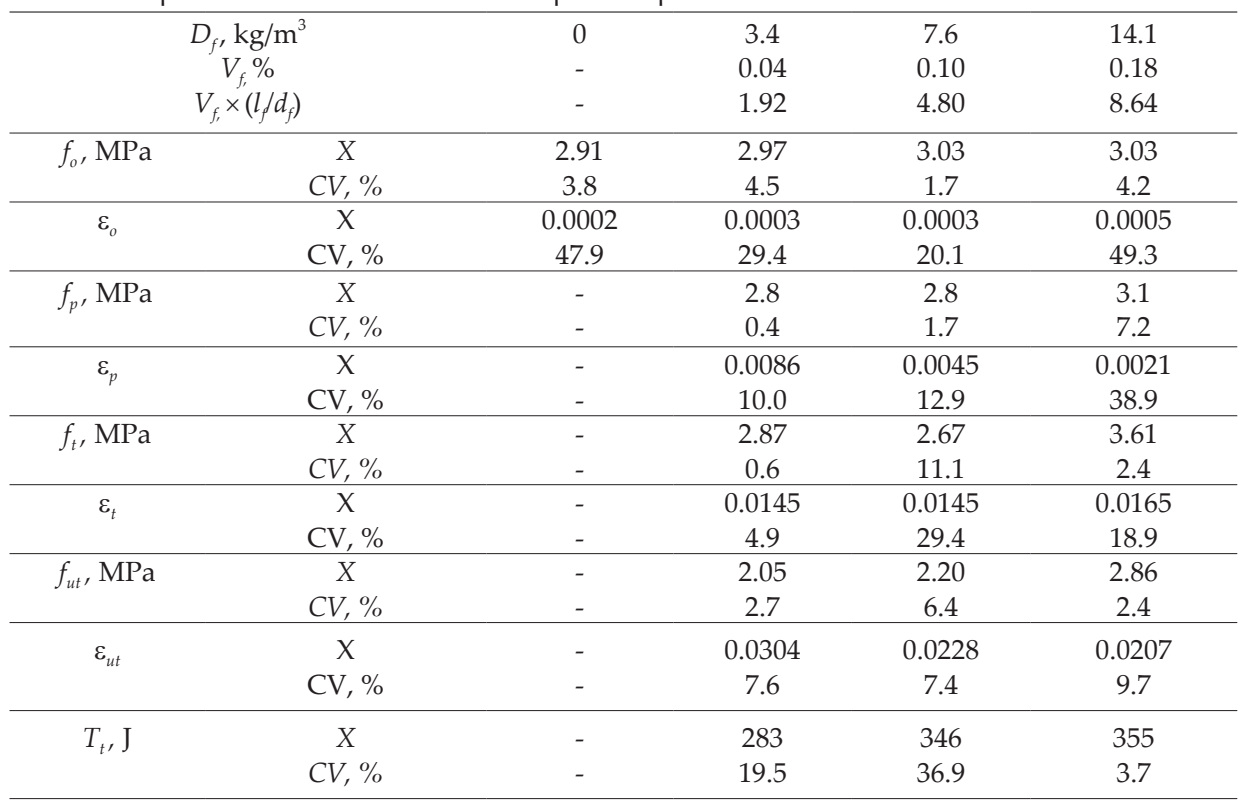
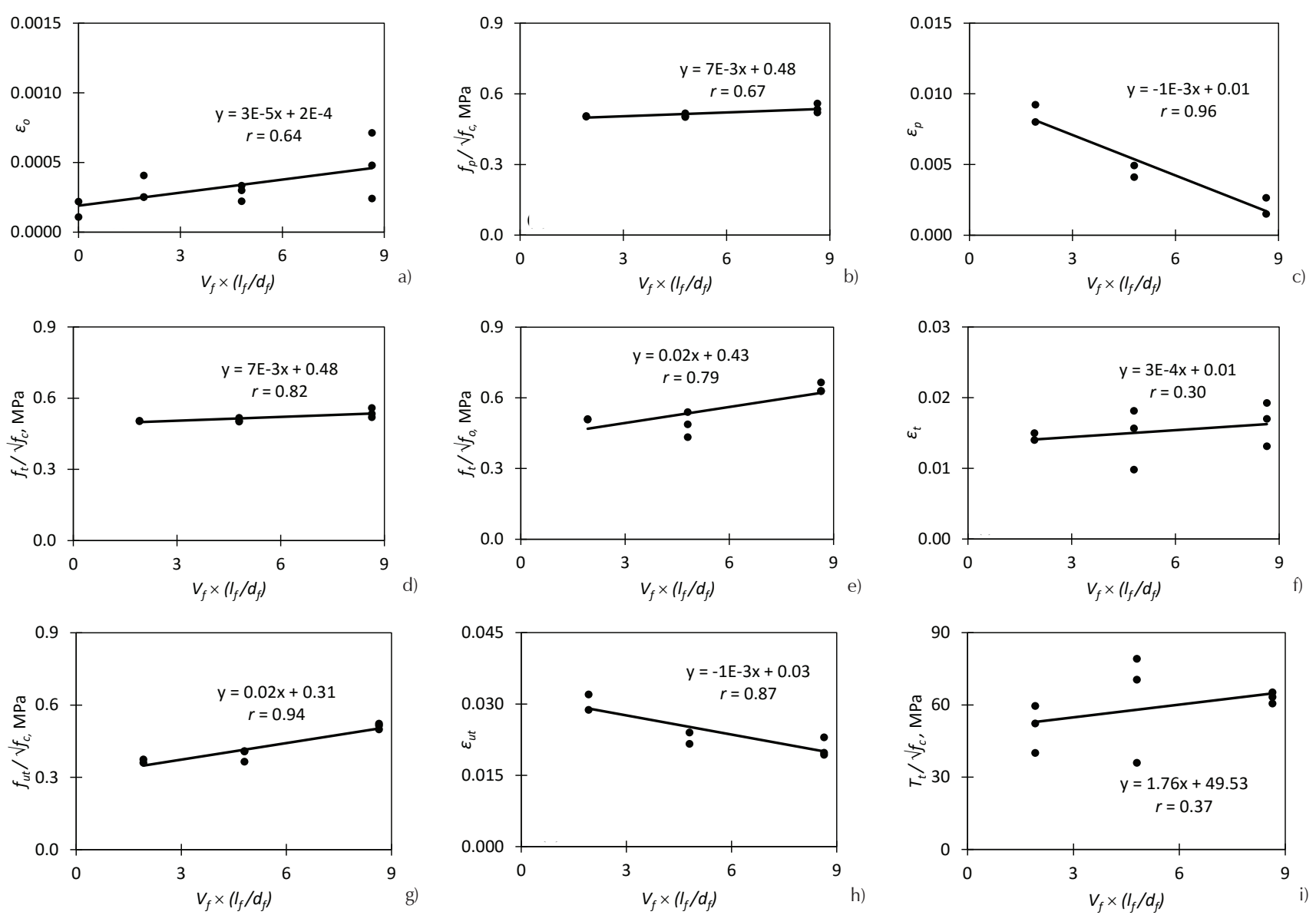

Figura 7. Tendencias de las propiedades mecánicas del concreto a tensión por compresión diametral del CRFA: a) $\varepsilon_{\alpha^{\prime}}$ b) $f_{p} / f_{c^{\prime}}$ c) $\varepsilon_{p^{\prime}}$ d) $f_{t} / f_{c^{\prime}}$ e) $f_{t} / f_{o^{\prime}}$ f) $\varepsilon_{t^{\prime}}$ g) $f_{u t} / f_{c}$, h) $\varepsilon_{u t}$. i) $T / \sqrt{ } f_{c}$. 

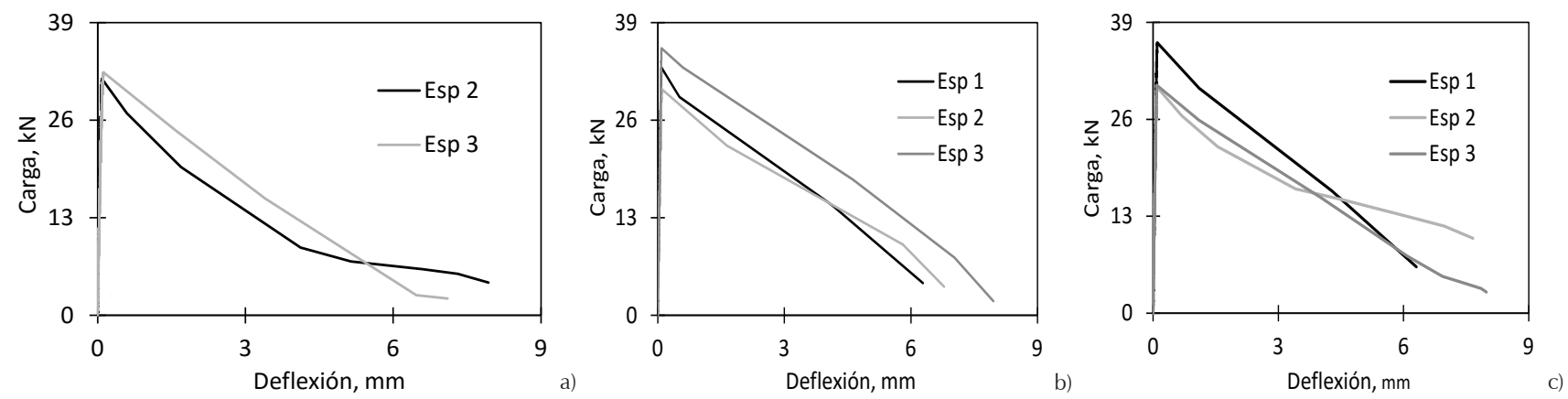

Figura 8. Curvas carga-deflexión del concreto en tensión por flexión: a) $D_{f}=3.4 \mathrm{~kg} / \mathrm{m}^{3}$, b) $D_{f}=7.6 \mathrm{~kg} / \mathrm{m}^{3}$, c) $D_{f}=14.1 \mathrm{~kg} / \mathrm{m}^{3}$

Tabla 3. Propiedades mecánicas del concreto a tensión por flexión

\begin{tabular}{|c|c|c|c|c|c|}
\hline \multicolumn{2}{|c|}{ Nomenclatura } & CS & CF-5 & CF-9 & CF-18 \\
\hline \multirow{3}{*}{\multicolumn{2}{|c|}{$\begin{array}{c}D_{f}, \mathrm{~kg} / \mathrm{m}^{3} \\
V_{f} \% \\
V_{f,} \times\left(l_{f} / d_{f}\right)\end{array}$}} & 0 & 3.4 & 7.6 & 14.1 \\
\hline & & - & 0.04 & 0.10 & 0.18 \\
\hline & & - & 1.92 & 4.80 & 8.64 \\
\hline \multirow{2}{*}{$f_{r}=f_{\max }, \mathrm{MPa}$} & $X$ & 4.2 & 4.2 & 4.2 & 4.3 \\
\hline & $C V, \%$ & 1.4 & 0.3 & 3.7 & 7.9 \\
\hline \multirow{2}{*}{$\delta_{r^{\prime}} \mathrm{mm}$} & X & 0.09 & 0.09 & 0.10 & 0.10 \\
\hline & $C V, \%$ & 2.7 & 2.4 & 17.9 & 4.7 \\
\hline \multirow{2}{*}{$f_{l c / 300} / f_{r}$} & $X$ & - & 0.72 & 0.77 & 0.78 \\
\hline & $C V, \%$ & - & 10.5 & 0.9 & 3.7 \\
\hline \multirow{2}{*}{$f_{l c / 150} / f_{r}$} & $X$ & - & 0.49 & 0.62 & 0.63 \\
\hline & $C V, \%$ & - & 14.1 & 7.8 & 6.6 \\
\hline \multirow{2}{*}{$f_{u^{\prime}} \mathrm{MPa}$} & $X$ & - & 0.44 & 0.46 & 0.85 \\
\hline & $C V, \%$ & - & 46.9 & 32.1 & 58.9 \\
\hline \multirow{2}{*}{$\delta_{u^{\prime}} \mathrm{mm}$} & $X$ & - & 7.4 & 7.4 & 7.8 \\
\hline & $C V, \%$ & - & 6.5 & 11.1 & 2.9 \\
\hline \multirow{2}{*}{$T_{f,} \mathrm{~J}$} & $X$ & 1.6 & 112.1 & 125.9 & 131.0 \\
\hline & $\mathrm{CV}, \%$ & 11.0 & 2.7 & 17.9 & 2.6 \\
\hline
\end{tabular}

tes a $l_{c} / 300$ y $l_{c} / 150$ incrementan ligeramente porque aumenta el contenido de fibras de acero. Los cocientes $f_{l c / 300} / f_{r}$ y $f_{l c / 150} / f_{r}$ no cumplen con lo indicado en la sección C5.6.6.2 de la NSR-10. Esto se debe a que se utilizaron bajas dosificaciones de fibras de acero; por ejemplo, inferiores a $60 \mathrm{~kg} / \mathrm{m}^{3}$, que es la dosificación mínima especificada en la NSR-10 para vigas. Los leves incrementos de resistencia y tenacidad se deben a los bajos contenidos de fibras de acero utilizados en el estudio.

\section{Desempeño a flexión de losas}

En la figura 10 se muestran las curvas carga-deflexión medidas durante los ensayos a flexión de las losas de CS y CRFA. La deflexión corresponde a la medida en el centro del claro de la losa. En la tabla 4 se observan los principales parámetros medidos en el ensayo a flexión de las de losas de CS y de CRFA. En la tabla se incluyen los parámetros de carga y deflexión asociados a la carga máxima $\left(P_{\max }, \delta_{\max }\right)$ y a la carga última $\left(P_{u}, \delta_{u}\right)$. El punto último se define para el momento en el cual se observó una pérdida abrupta de la capacidad de resistencia. En la figura 10 y en la tabla 4 se observa que la carga $P_{\max }$ no varió significativamente con el incremento de la dosificación de fibras de acero. Khalloo y Afshari (2005) y Sorelli et al. (2004) también observaron que en las losas con CRFA, la fuerza última a flexión no incrementa significativamente cuando las fibras se agregan al concreto. Sin embargo, se observa que la deflexión en $P_{\max }$ $\left(\delta_{\max }\right)$ y la deflexión en $P_{u}\left(\delta_{u}\right)$ aumentaron de acuerdo con el incremento del producto $\left[V_{f} \times\left(l_{f} / d_{f}\right)\right]$. Hadi (2008) también observó que la deflexión última aumenta en las losas de CRFA. 

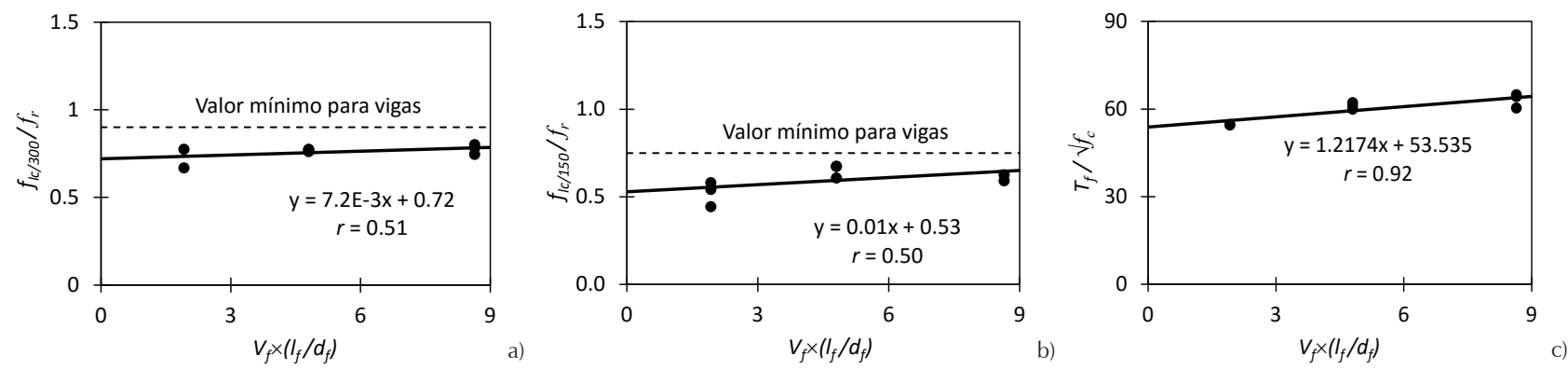

Figura 9. Tendencias de las principales propiedades mecánicas del CRFA en tensión por flexión

Tabla 4. Parámetros principales del ensayo a flexión de losas

\begin{tabular}{cccccc}
\hline \multicolumn{2}{c}{ Nomenclatura } & CS & CF-5 & CF-9 & CF-18 \\
\hline \multicolumn{2}{c}{$D_{f}, \mathrm{~kg} / \mathrm{m}^{3}$} \\
$V_{f,} \%$ \\
$V_{f,} \times\left(l_{f} / d_{f}\right)$
\end{tabular}
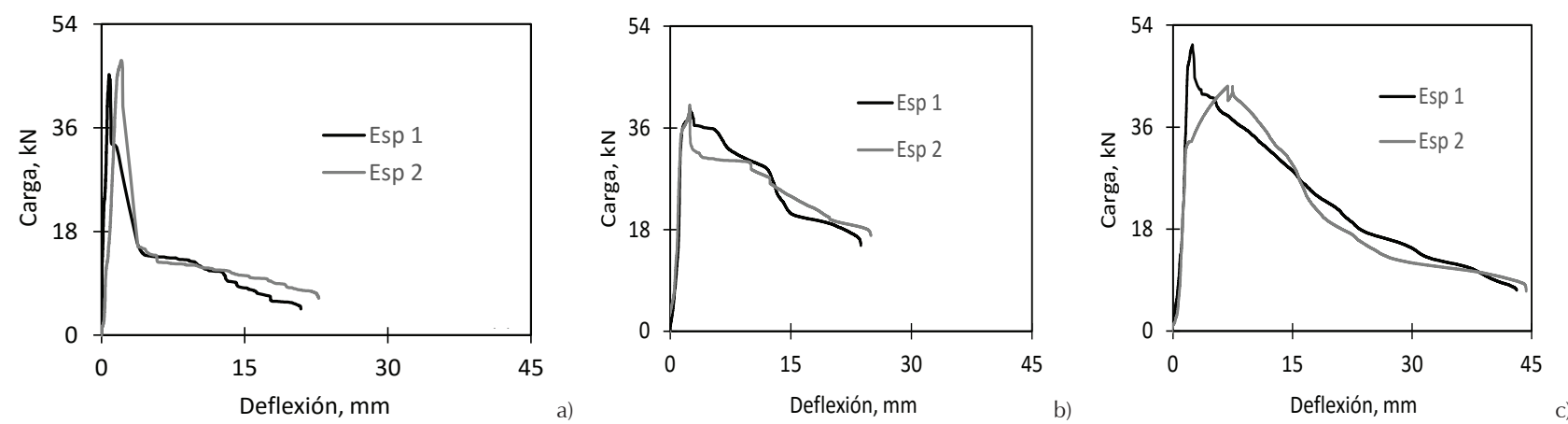

Figura 10. Curvas carga-deflexión de losas de CRFA: a) $D_{f}=3.4 \mathrm{~kg} / \mathrm{m}^{3}$, b) $D_{f}=7.6 \mathrm{~kg} / \mathrm{m}^{3}$, c) $D_{f}=14.1 \mathrm{~kg} / \mathrm{m}^{3}$

La absorción de energía o tenacidad representa el área bajo la curva carga-deflexión y se determinó por medio del método de los trapecios. En la figura 11 se muestra la variación de la energía en función de la deflexión en el centro de la luz de las losas. En la tabla 4 se indican los valores promedio de la energía para el momento de la falla de la losa, $T_{l}$. En la figura se observa que a medida que aumenta la dosificación de fibras de acero, la absorción de energía aumenta, debido a que las fibras generan un mayor número de micro-fisuras y un efecto de "enganche" que permite que se generen anchos de grietas significativos antes de que se presente la falla por extracción de las fibras. Khalloo y Afshari (2005), Sorelli et al. (2004) y Hadi (2008) también observaron que en las losas con CRFA se obtiene un comportamiento más estable (poca degradación) y la capacidad de absorción de energía mejora significativamente. 


\section{Conclusiones}

En este artículo se presentaron y discutieron los resultados de un programa experimental para estudiar el desempeño de losas de concreto sobre terreno reforzadas con fibras de acero en dosificaciones de 5.9 y $18 \mathrm{~kg} / \mathrm{m}^{3}$, como alternativa a las losas de contrapiso para vivienda reforzadas con malla electrosoldada. Los ensayos de caracterización de las propiedades mecánicas del material demostraron que el módulo de elasticidad disminuye cuando incrementa la dosificación de fibras, debido a una disminución de la cantidad de agregado grueso por la presencia de las fibras. También se observó una disminución del coeficiente de Poisson debido al confinamiento que las fibras generan en la matriz. En cuanto a la deformación asociada a la resistencia máxima a compresión se observó que este valor fue, en promedio, 56\% mayor al observado en el concreto simple, debido al efecto de "enganche" de las fibras en la matriz del concreto; es decir, mayor capacidad de deformación asociada a la resistencia. En los ensayos de tensión por compresión diametral se observó que la resistencia máxima a tensión por compresión diametral incrementan al aumentar la dosificación de fibras, ya que en ese punto las fibras proporcionan al concreto no solo la máxima capacidad de resistencia, sino también una capacidad de deformación a tensión mayor que la que se presenta en un concreto sin fibras. En los ensayos de tensión por flexión se observó que las resistencias residuales y la tenacidad aumentaron levemente de acuerdo con el incremento del contenido de fibras de acero, debido a que se utilizaron bajas dosificaciones de fibras de acero; por ejemplo, inferiores a $60 \mathrm{~kg} / \mathrm{m}^{3}$, que es la dosificación mínima especificada en la NSR-10 para vigas.

Durante los ensayos a flexión se observó que las losas de concreto simple colapsaron súbitamente y experimentaron poca capacidad de absorción de energía. Este comportamiento se podría traducir en losas sobre terreno que son inestables y con poca durabilidad durante su vida útil. Las curvas carga-deflexión y las curvas tenacidad-deflexión demostraron que, a diferencia de la carga máxima de las losas, las deflexiones en las cargas máxima y última, y la tenacidad en la falla aumentaron significativamente al incrementar la dosificación de fibras de acero. La utilización de fibras de acero en losas de concreto sobre terreno en viviendas con cargas menores a $500 \mathrm{~kg} / \mathrm{m}^{2}$, promueve un comportamiento más dúctil de las losas y podrían aumentar la capacidad de resistencia del elemento con una determinada dosificación. Los resultados de este estudio pueden utilizarse para estimar, en estudios futuros, la dosificación de fibras necesaria para obtener un comportamiento a flexión equivalente al de la losa de concreto reforzada convencionalmente con malla electrosoldada. A pesar de que se requieren estudios complementarios para comparar el comportamiento de las losas de concreto reforzadas con fibras de acero o con refuerzo convencional (malla electrosoldada), es importante que se promueva el uso de fibras de acero en losas sobre terreno para viviendas, no solo para aumentar la calidad de los materiales, sino también para disminuir los costos e incrementar la durabilidad de este tipo de elementos.

\section{Agradecimientos}

Los autores agradecen a la Vicerrectoría de Investigaciones de la Universidad Militar Nueva Granada (UMNG, Colombia) por el financiamiento del proyecto ING-1536. Adicionalmente, los autores expresan su agradecimiento a las empresas Argos y Proalco (representante de Bekaert en Colombia), en especial a los ingenieros Diego Velandia y Liliana Cardona. De la misma forma, al ingeniero Felipe Riveros por su apoyo en la realización de las pruebas en los Laboratorios de Agregados y Concretos de la UMNG.

\section{Referencias}

ACI-Committee 544. State of the art report on fiber reinforced concrete (ACI 544.1R-96). American Concrete Institute, Farmington Hills, MI, USA, 1996.

ACI-Committee 544. Report on the physical properties and durability of fiber-reinforced concrete (ACI 544.5R-10). American Concrete Institute, Farmington Hills, MI, USA.

Asociación Científico-Técnica del Hormigón Estructural, 2010.

ACHE. Manual de tecnología del hormigón reforzado con fibras de acero, Madrid, España, 2000.

ASTM-C-1609. Standard test method for flexural performance of fiberreinforced concrete (using beam with third-point loading), Libro annual, USA, 2012.

Carrillo J., Riveros F., LLano L. Dispositivo para sujeción de múltiples transductores de desplazamiento en ensayos de losas de concreto. Ingeniería Mecánica, Tecnología y Desarrollo, volumen 5 (número 2), 2015: 313-319.

Carrillo J., Barrera P. y Acosta A. Evaluación del desempeño a tensión por compresión diametral del concreto reforzado con fibras de acero ZP-306. Ingeniería y competitividad, volumen 16 (número 1), 2014: 262-272.

Carrillo J., Gonzalez G., Aperador W. Correlaciones entre las propiedades mecánicas del concreto reforzado con fibras de acero. Ingeniería, investigación y tecnología, volumen 14 (número 3), 2013: 435-450. 
European Standard Norme Européenne Europäische Norm, EN14488-5. Testing sprayed concrete - Part 5: Determination of energy absortion capacity of fiber reinforced slab specimens, Comité Europeen de Normalisation (CEN), Bruselas, Bélgica, 2006.

Gallo L., Gonzales G., Carrillo J. Comportamiento del concreto reforzado con fibras de acero ZP-306 sometidos a esfuerzos de compresión. Ciencia e Ingeniería Neogranadina, volumen 23 (número 1), 2013: 117-133.

Hadi M. An investigation of the behavior of steel and polypropylene fiber reinforced concrete slabs, en: 7th International Conference: Concrete, Construction's sustainable option, Escocia, 2008.

Hannant D. Fiber cements and fiber concretes. Journal of Polymer Science, volumen 17 (número 7), 1978: 464-465.

Khalloo A. y Afshari M. Flexural behavior of small steel fiber reinforced concrete slabs. Cement and concrete Composites, volumen 27 (número 1), 2005: 141-149.

Mármol P. Hormigones con fibras de acero características mecánicas (tesis de maestría), Universidad Politécnica de Madrid, España, 2010.

NTC 673. Ensayo de resistencia a la compresión de especímenes cilíndricos de concreto, ICONTEC, Bogotá, Colombia, 2010.

NTC 722 Ensayo de resistencia a la tensión indirecta de especímenes cilíndricos de concreto, ICONTEC, Bogotá, Colombia, 2000.

NTC 4025 Método de ensayo para determinar el módulo de elasticidad estático y la relación de Poisson en concreto a compresión, ICONTEC, Bogotá, Colombia, 2006.

NTC 5721. Método de ensayo para la determinación de la capacidad de absorción de energía (Tenacidad) de concreto reforzado con fibra, ICONTEC, Bogotá, Colombia, 2009.

NSR-10. Reglamento Colombiano de Construcción Sismo Resistente, Asociación Colombiana de Ingeniería Sísmica (AIS), Bogotá, Colombia, 2010.
Sika Colombia S.A. Antisol blanco: Curador para concreto y mortero en ambiente normal, Tocancipa, Colombia, 2015.

Sorelli L., Meda A., Plizzari G., Rossi B. Experimental investigation on slabs on grade: Steel fibers vs conventional reinforcement, 6th International RILEM Symposium on Fibre Reinforced Concretes, Varenna, Italy, 2004, pp. 1083-1092.

Yazici S. Effect of aspect ratio and volume fraction of steel fiber on the mechanical properties of SFRC. Construction and Building Materials, volumen 21 (número 6), 2007: 1250-1253.

\section{Este artículo se cita:}

\section{Citación estilo Chicago}

Carrillo, Julián, Diego Silva-Páramo. Ensayos a flexión de losas de concreto sobre terreno reforzadas con fibras de acero. Ingeniería Investigación y Tecnología, XVII, 03 (2016): 317-330.

\section{Citación estilo ISO 690}

Carrillo J., Silva-Páramo D.S. Ensayos a flexión de losas de concreto sobre terreno reforzadas con fibras de acero. Ingeniería Investigación y Tecnología, volumen XVII (número 3), julio-septiembre 2016: 317-330.

\section{Semblanzas de autores}

Julián Carrillo. Obtuvo los títulos de ingeniero civil en 2002 por la Universidad Militar Nueva Granada, UMNG, Colombia, el de maestro en ingeniería civil (área de estructuras y sísmica) en 2004 por la Universidad de los Andes, Colombia, y el de doctor en ingeniería (área de estructuras) en 2010 por la Universidad Nacional Autónoma de México, UNAM. Actualmente es profesor asociado y director del grupo de investigación de estructuras y sísmica de la UMNG. Es miembro de tres comités del American Concrete Institute, ACI: 314, Diseño simplificado de edificios; 369, Reparación y rehabilitación sísmica; y 374, Diseño sísmico basado en desempeño de edificios de concreto.

Diego Silva-Páramo. Obtuvo el título de ingeniero civil en 2015 por la Universidad Militar Nueva Granada, UMNG, Colombia. Actualmente es auxiliar de investigación del grupo de investigación de estructuras y sísmica de la UMNG. 